\title{
Quenching 2D Quantum Gravity
}

\author{
C.F. Baillie \\ Dept of Computer Science, University of Colorado \\ Boulder, CO 80309, USA \\ K.A. Hawick \\ Northeast Parallel Architecture Center \\ Syracuse University \\ 111 College Place \\ Syracuse, NY 13244, USA \\ D.A. Johnston \\ LPTHE \\ Universite Paris Sud, Batiment 211 \\ F-91405 Orsay, France ${ }^{1}$
}

November 13, 2018

\begin{abstract}
We simulate the Ising model on a set of fixed random $\phi^{3}$ graphs, which corresponds to a quenched coupling to $2 \mathrm{D}$ gravity rather than the annealed coupling that is usually considered. We investigate the critical exponents in such a quenched ensemble and compare them with measurements on dynamical $\phi^{3}$ graphs, flat lattices and a single fixed $\phi^{3}$ graph.
\end{abstract}

Submitted to Phys Lett B.

1 Address: Sept. 1993 - 1994, Permanent Address: Maths Dept, Heriot-Watt University, Edinburgh, Scotland 
It has recently become possible to calculate the critical exponents for various spin models on a particular class of dynamical connectivity lattices using both the methods of conformal field theory [1] and matrix models [2]. Remarkably, it is even possible to solve the Ising model exactly in the presence of an external field on such a lattice, which has proven impossible on fixed 2D lattices [3]. The key to the calculations, whether in the continuum conformal field theory formalism or the discrete matrix model formalism, lies in the observation that putting the model on a dynamical lattice and allowing it to interact with the lattice is equivalent to coupling the model to $2 \mathrm{D}$ quantum gravity. This has the effect of "dressing" the conformal weights $\Delta_{0}$ of the critical continuum theory on a flat $2 \mathrm{D}$ lattice, to give new conformal weights $\Delta$

$$
\Delta-\Delta_{0}=-\frac{\xi^{2}}{2} \Delta(\Delta-1)
$$

where

$$
\xi=-\frac{1}{2 \sqrt{3}}(\sqrt{25-c}-\sqrt{1-c})
$$

and $c$ is the central charge of the theory in question. This, in turn, will effect the critical exponents of theory. These can be calculated from the conformal weights of the energy and spin operators $\Delta_{\epsilon}$ and $\Delta_{\sigma}$ respectively [⿶ which give $\alpha$ (the specific heat exponent) and $\beta$ (the magnetization exponent)

$$
\begin{aligned}
\alpha & =\frac{1-2 \Delta_{\epsilon}}{1-\Delta_{\epsilon}} \\
\beta & =\frac{\Delta_{\sigma}}{1-\Delta_{\epsilon}}
\end{aligned}
$$

in the usual fashion. Given $\alpha$ and $\beta$ from the conformal field theory we can then use the various standard scaling relations to calculate the full array of exponents. For the exactly soluble case of the Ising model on a dynamical lattice the standard scaling relations can be shown to hold, so it is not too great a leap of faith to assume their validity for other models.

There is a growing body of numerical evidence confirming the new dynamical exponents in simulations [5, 6, 7], both on dynamical triangulations and their dual dynamical $\phi^{3}$ graphs with both spherical and toroidal topology lattices 1 . The matrix model approach suggests that any dynamical polygonalization of a surface should give the same exponents for a given model, so there should be universality in this sense. If we think of the numerical simulations purely as an exercise in statistical mechanics it is clear they represent taking an annealed average over the various random graphs on which the spin models live. The partition function of the Ising model on dynamical lattices, for instance, is

$$
Z=\sum_{G} \sum_{\{\sigma\}} \exp \beta\left(\sum_{<i j>} G_{i j} \sigma_{i} \sigma_{j}+H \sum_{i} \sigma_{i}\right)
$$

where the connectivity matrix of the lattice is $G_{i j}$. The dynamics of the lattice, represented by the sum over random $\phi^{3}$ graphs or their dual triangulations $\sum_{G}$, takes place on the same timescale as the dynamics of the spins, which is manifest in the numerical simulations where the local "flip" moves that change the lattice connectivity to perform the sum $\sum_{G}$ are mixed in with spin cluster updates. An interesting related question, to which the answer is not immediately clear, is what exponents would be measured in a simulation on a fixed 2D quantum gravity graph.

A first reflex is to guess that with a fixed lattice we would find the Onsager exponents. This appears to be correct when we consider random lattices with "weak" disorder - ie sufficiently close to regular 2D lattices, where the Harris criterion (roughly paraphrased as "weak disorder has no effect if $\alpha<0$ ") gives the Ising model as a marginal case, and simulations come down on the side of Onsager exponents [8] 2 . Other fixed random lattices which are close in some sense to flat lattices $\beta$, such as Voronoi lattices also

\footnotetext{
${ }^{1}$ The topology of the underlying lattice is not expected to affect the spin model critical exponents, though it does have an effect on the exponent $\gamma_{\text {string }}$.

${ }^{2}$ The analytical work of the Dotsenkos 9, however, suggests that randomness in the bond values can weaken the specific heat singularity.

${ }^{3}$ Having a fractal dimension of 2 , for instance.
} 
give Onsager exponents [10]. There has been one simulation to date on a single random $\phi^{3}$ graph [11] generated by the Tutte algorithm which proved an efficient means of producing large pure (ie no matter) 2D gravity lattices [12], that found results that appeared to be compatible with the Onsager exponents. A moment's reflection suggests that this is a rather surprising result - the fractal dimension of 2D gravity coupled to conformal matter with central charge $c$ has been calculated [13], and modulo some caveats about lattice dependent effects on the lower moments of the density of nodes [14], is given by

$$
D=\left(1+\frac{\sqrt{13-c}}{\sqrt{25-c}}\right) \frac{\gamma Q}{2}
$$

where $Q=\sqrt{(25-c) / 3}$ and $\gamma=\left(Q-\sqrt{Q^{2}-8}\right) / 2$. We find that $D$ for $c=1 / 2$, the Ising model interacting with a dynamical lattice, is equal to 3 and $D$ for $c=0$, ie pure $2 \mathrm{D}$ gravity, is $(5+\sqrt{13}) / 3 \simeq$ $2.8685 \ldots$ We would thus expect to find $d \simeq 2.8685$ for the graph used in [1], which is very different from the 2 of a flat lattice. Unfortunately, we can't deal analytically with a single fixed $\phi^{3}$ graph to see what exponents we might expect, but we can aspire to taking a quenched average over a set of different fixed $\phi^{3}$ graphs.

It is often the case in statistical mechanics that quenched averages, in which the disorder is frozen in on the timescale of the spin dynamics, are more interesting than annealed averages, particularly in systems where frustration is present such as spin-glasses [15. All of the couplings are positive in the model of equ.(4), so we have no frustration and hence would not expect to see any of the rich behaviour of [15], but it is still interesting to ask what happens to the critical exponents of the model when we consider a quenched sum over the random graphs rather than an annealed sum. In a simulation we would implement such a quenched summation by simulating our Ising model on an appropriate set of fixed random graphs and then taking a cross lattice average to obtain our observables, as is done in the simulation of spin glasses to average over different sets of spin couplings. In 16 it was suggested that applying the replica trick 15] in conjunction with the continuum methods of [1] allowed the calculation of the exponents in a quenched average over 2D gravity graphs. For a quenched summation the free energy $F$ is calculated on each graph before summing over the random graphs

$$
F=\sum_{G} \log Z(G)
$$

where $Z(G)$ is the partition function for the Ising model on a particular random graph

$$
Z(G)=\sum_{\{\sigma\}} \exp \beta\left(\sum_{<i j>} G_{i j} \sigma_{i} \sigma_{j}+H \sum_{i} \sigma_{i}\right) .
$$

The awkward logarithm inside the sum can be dealt with by replacing $\log Z \simeq\left(Z^{n}-1\right) / n, n \rightarrow 0$ to give a more familiar partition function, now with $n$ replicas of the original matter. Observables are calculated with this replica partition function and the limit $n \rightarrow 0$ is taken in order to obtain the desired quenched averages. Translating this approach directly to the continuum language of [1], we can obtain the quenched value of the one point function $F_{\Phi}(A)$ of an operator $\Phi$, the magnetization or the energy for instance, by inserting one copy $\Phi^{a}$ of $\Phi$ into the fixed intrinsic area partition function $Z_{n}(A)$ for $n$ copies of the matter theory

$$
\begin{aligned}
F_{\Phi}(A) & =\frac{\int D \phi D X \exp \left(-S^{n}\right) \delta\left(\int e^{\xi \phi} \sqrt{\hat{g}} d^{2} z-A\right) \int \Phi^{a} e^{\rho \phi} \sqrt{\hat{g}} d^{2} z}{Z_{n}(A)} \\
n \rightarrow 0 &
\end{aligned}
$$

where the action $S^{n}$ is composed of a Liouville part and a Feigen-Fuchs action [17 for $n$ matter copies

$$
S_{M}^{n}=\frac{1}{2 \pi} \sum_{a=1}^{n} \int d^{2} z\left(\partial X^{a} \bar{\partial} X^{a}+\frac{i}{2} \alpha_{0} \sqrt{\hat{g}} \hat{R} X^{a}\right)
$$


and $\alpha_{0}$ is determined by $c=1-12 \alpha_{0}^{2}$. Applying the standard scaling arguments of DDK [1] to such a theory and taking the limit $n \rightarrow 0$ to get the quenched averages, we find the following values for the quenched conformal weights

$$
\Delta_{\text {quenched }}=\frac{ \pm \sqrt{1+24 \Delta_{0}}-1}{4} .
$$

If we plug in the values for the energy and spin operators in the Ising model we find the following quenched weights

$$
\begin{aligned}
\Delta_{\epsilon} & =\frac{\sqrt{13}-1}{4} \simeq 0.6513878 \\
\Delta_{\sigma} & =\frac{\sqrt{5 / 2}-1}{4} \simeq 0.1452847,
\end{aligned}
$$

which, using equ.(3), gives the following values for the critical exponents $\alpha$ and $\beta$

$$
\begin{aligned}
& \alpha=\frac{6-2 \sqrt{13}}{5-\sqrt{13}} \simeq-0.8685170 \\
& \beta=\frac{\sqrt{5 / 2}-1}{5-\sqrt{13}} \simeq 0.4167516 .
\end{aligned}
$$

Applying the scaling relations then gives the full set of quenched critical exponents, which we repeat from [16] in Table.1 below for completeness, along with the fixed lattice (Onsager) and annealed (dynamical lattice KPZ/DDK) exponents.

\begin{tabular}{|c|c|c|c|c|c|c|}
\hline Type & $\alpha$ & $\beta$ & $\gamma$ & $\delta$ & $\nu$ & $\eta$ \\
\hline Fixed & 0 & $\frac{1}{8}$ & $\frac{7}{4}$ & 15 & 1 & $\frac{1}{4}$ \\
\hline Annealed & -1 & $\frac{1}{2}$ & 2 & 5 & $\frac{3}{d}$ & $2-\frac{2 d}{3}$ \\
\hline Quenched & -0.8685169 & 0.4167516 & 2.0350137 & 5.8830375 & $\frac{2.8685169}{d}$ & $2-0.7094306 d$ \\
\hline
\end{tabular}

Table 1: Critical exponents for Ising models

We thus predict a set of quenched exponents that are close, but not equal to, the dynamical lattice exponents. The non-rational exponents might be a little disquieting at first site, but they seem to be a consequence of forcing the model to live in a gravitational background with the "wrong" value of $c$ (ie $c=0$ effectively as we have a total central charge of $n c, n \rightarrow 0$ in the quenched theory rather than the natural $c=1 / 2$ ). Similar non-rational exponents were calculated and observed for percolation, where the natural value of $c$ is eaual to zero, on $c \neq 0$ string worldsheets in [18]. In fact if we insert the appropriate values for the fractal dimension calculated from equ.(5) into the above table we see that we obtain the same values (1, 0 respectively) for the exponents $\nu$ and $\eta$ in both the annealed and quenched cases. If we take these two exponents as our starting point and calculate the rest from the scaling relations, we could argue that the quenched model differs only from the annealed model in living in a slightly different dimension. We have not found the fixed lattice values for the exponents in the above calculation because switching off 2D gravity completely corresponds to the limit $c \rightarrow-\infty$ rather than $c \rightarrow 0$. The effects of 2D gravity are thus still visible, even in an ensemble of fixed $\phi^{3}$ graphs.

So much for the theory. The results of [11] suggest that the Onsager exponents appear on a fixed $\phi^{3}$ graph, so an average over a set of such exponents would still give Onsager exponents, in spite of the above arguments. In this paper we report on a simulation where we implement a quenched average explicitly by simulating $O(100)$ Ising models on different fixed $\phi^{3}$ graphs simultaneously using a parallel computer. We have resurrected and modified the program used in earlier simulations on dynamical lattices [5] for this purpose, so we perform a microcanonical (fixed number of nodes) simulation on $\phi^{3}$ graphs of spherical topology without tadpoles or self-energy bubbles with $N=124,250,500,1000$ and 2000 nodes. We used the Tutte algorithm to generate the one hundred or so $\phi^{3}$ graphs of each size that were required. We 
simulated at a range of $\beta$ values from 0.1 to 1.1, using hot and cold starts where appropriate, with $10 \mathrm{~K}$ metropolis thermalization sweeps and $50 \mathrm{~K}$ Wolff cluster algorithm update sweeps at each $\beta$ value and graph size. Note that the absence of $N F L I P=N$ dynamical flip moves between each spin update in the quenched simulation means that our statistics are rather more modest than the dynamical lattice statistics in [5]. The various observables were measured on each graph and a cross graph average taken to implement the quenched sum. The errors quoted are those coming from the average across the different graphs, rather than individual graph statistical errors.

The standard observables were measured: the energy $E$, specific heat $C$, magnetization $M$, susceptibility $\chi$, correlation length $\xi$ and Binder's cumulant for the magnetization

$$
U=1-\frac{<M^{4}>}{3<M^{2}>}
$$

along with the acceptance for the Wolff algorithm, to verify that an appropriate number of sweeps was being carried out. The number of Wolff sweeps was adjusted so that ( 1 / average size of Wolff cluster) sweeps were performed per measurement at each $\beta$. In our original dynamical lattice simulations in [5] we had some difficulty in finding a reliable estimate for the crossing point of Binder's cumulant for different lattice sizes, which is the usual means of locating the critical point with these methods, so we adopted an alternative approach that gave good results for the exactly soluble case of the Ising model, where the critical point is known analytically. The expected scaling behaviour for Binder's cumulant is $U \simeq U\left(t N^{1 / \nu D}\right)$, so its derivative with respect to $t$, or equivalently $\beta$, will scale as $1 / \nu D$ at $\beta_{c}$. We would also expect the maximum slope to scale in the same way

$$
\max \left(\frac{d U}{d \beta}\right) \simeq N^{1 / \nu D}
$$

which can be used to obtain $\nu D$. Knowing this the finite size scaling relation

$$
\left|\beta_{c}^{N}-\beta_{c}^{\infty}\right| \simeq N^{-1 / \nu D}
$$

with $\beta_{c}^{N}$ obtained from either the peak in the specific heat or the maximum slope in the cumulant, can be used to extract $\beta_{c}^{\infty}$. In Fig. 1 we show the maximum slope of the cumulant plotted against $N$ on a logarithmic scale along with the fitted line with slope $1 / \nu D=0.32(1)$. We thus have $\nu D=3.1(1)$, which can then be fed into the finite size scaling relation in equ.(15) to get $\beta_{c}^{\infty}$. In Fig. 2 we show the results for both the maximum slope in the cumulant and the peak in the specific heat, giving $\beta_{c}^{\infty}=0.778(2)$, where we have dropped the smallest lattice size from the fit to the cumulant slope. We also found that it was possible to extract $\beta_{c}$ from the crossing of cumulants for different lattice sizes with the current data, which gives an additional estimate of $\beta_{c}=0.78(1)$ compatible with the value above, as is the corresponding estimate of $\nu D$ with this method.

The scaling relation $\alpha=2-\nu D$ thus gives $\alpha=-1.1(1)$ for the specific heat critical exponent, which is a long way from the logarithmic scaling $(\alpha=0)$ given by the Onsager exponents. It is also possible to attempt to extract $\alpha$ directly from the singular behaviour of the specific heat

$$
C \simeq B+C_{0} t^{-\alpha}
$$

or from finite size scaling

$$
C \simeq B^{\prime}+C_{0}^{\prime} N^{\alpha / \nu D} .
$$

Using the singular behaviour proves to be hopeless with our data as essentially any value for $\alpha$ can be obtained with comparable $\chi^{2} \mathrm{~s}$, but the finite size scaling fit gives a minimum $\chi^{2}$-fit for $B^{\prime}=1.8$ and $\alpha / \nu D=-0.37(1)$, which gives $\alpha=-1.1(1)$, in good agreement with the value obtained indirectly from the scaling relation.

A measurement of the exponent $\gamma$ governing the divergence of the susceptibility is less useful in distinguishing between the different sets of exponents as the quenched and annealed values are essentially identical $(\simeq 2)$ and the Onsager value is close to these (1.75). A fit to

$$
\chi=\chi_{0} t^{-\gamma}
$$


gives $\gamma=2.1(2)$ on the $N=2000$ lattices and $\gamma=2.0(2)$ for $N=1000$, which although it favours the dynamical/quenched values, is not really sufficient to exclude the Onsager values. The finite size scaling fit gives a much poorer fit with an even larger value of $\gamma$. In Fig. 3 we plot the divergence in $\chi$ for the two lattice sizes along with lines of slope 1.75 and 2 . It is clear from the diagram that it is indeed not really possible to decide conclusively between the candidate values from the data. Fitting $\beta$ is also rather unilluminating, whether by using $M \simeq M_{0} t^{\beta}$ or $M \simeq M_{0}^{\prime} N^{-1 / \nu D}$ as we find values of $0.2-0.3$ stranded in no man's land between Onsager and dynamical/quenched exponents. We found in [5] that the fits to $\beta$ on dynamical lattices were the poorest, so a similar result here is not surprising. It is not clear why the fit to the magnetic exponent should be so poor, but it was pointed out in [7] that the dynamical critical exponent $z$ for the magnetization was much larger than that for the energy on dynamical lattices (with the Wolff algorithm) possibly because of clusters becoming trapped in "bottlenecks" in the graph geometry. It is therefore conceivable that one has to work harder to overcome autocorrelation and finite size effects in the measurements of $\beta$ than for other exponents, whether on dynamical or quenched 2D gravity graphs.

We can also fit the exponent $\nu$ by looking at the divergence of the correlation length $\xi$ at $\beta_{c}$. As this involves a double fit, first for the correlation length

$$
C_{i j}=<\frac{1}{n(r)} \sum_{i j} \sigma_{i} \sigma_{j} \delta\left(d_{i j}-r\right)>\simeq \exp \left(-\frac{d_{i j}}{\xi}\right)
$$

(where $d_{i j}$ is the geodesic distance on the lattice between points $i$ and $j$ and $n(r)=\sum_{i j} \delta\left(d_{i j}-r\right)$ ) and then to the divergence in $\xi$, the fit is not likely to be very accurate. Doing this however, we find $\nu=0.87(2)$ for the data on the $N=2000$ graphs, which is identical to value of $\nu$ we measured on dynamical graphs in [5]. If we take the theoretical values for $D$ for both the quenched and annealed graphs we would expect $\nu=1$, just as for the Onsager exponents.

In summary, the fits described above are thus compatible with both those in [5] on dynamical $\phi^{3}$ graphs and with the similar quenched exponents. The best results are for $\nu D$ and $\alpha / \nu D$, both of which give a cusp $(\alpha=-1.1(1))$ in the specific heat, rather than a logarithmic divergence, as expected for a flat 2D lattice. The measured value of $\gamma$ on the largest lattices used, 2.1, is close to the $\simeq 2$ expected on dynamical/quenched graphs, but the Onsager value of 1.75 lies not far outside the error bars. The measured value of $\nu$ is identical to that found for dynamical graphs, but one cannot use $\nu$ to distinguish between Onsager and dynamical exponents. The values of $\beta$ measured are as disastrous as those found in [5] on dynamical lattices, and hence not very useful. Finally, it is worth noting that the measured value of $\beta_{c}^{\infty}$ in the quenched simulation is close to the calculable $\beta_{c}$ on dynamical graphs (0.7733185).

Although it would be dangerous to claim that there was conclusive evidence for non-Onsager exponents in the quenched ensemble on the basis of fits to $\nu D$ and $\alpha$ alone, the data for these is at least consistent with this, and none of the other fits contradict it. Unfortunately, the results are nowhere near accurate enough to distinguish between the postulated quenched exponents and dynamical graph exponents, even with the $O(100)$ different graphs used. If we accept that the quenched ensemble does give non-Onsager exponents we are left with the task of explaining the results of [11] on a single 2D gravity graph, where acceptable fits were found to the Onsager values. It is possible that taking the continuum limit in two different ways, on a fixed graph and in an ensemble of fixed graphs, simply gives different exponents, though as one expects self-averaging for quantities such as the energy and magnetization it is difficult to see how this might happen. Another possibility is that the particular random graph chosen in 11 . produced a set of pseudo-Onsager exponents by chance, as there appears to be a wide variation in the apparent critical points and exponents if these are fitted in the individual graphs in our ensemble. Earlier simulations on fixed random lattices, of the XY model in particular 19], have shown that finite size effects can be particularly important too. In closing it is amusing to note that, formally at any rate, we would expect to see the same quenched exponents appear in a simulation of an Ising model on a single dynamical graph in which the back reaction of the spins on the lattice was switched off (by always carrying out flips rather than performing a Metropolis test on the resulting energy change) as this also has $c=0$.

This work was supported in part by NATO collaborative research grant CRG910091. CFB is supported by DOE under contract DE-FG02-91ER40672, by NSF Grand Challenge Applications Group Grant ASC- 
9217394 and by NASA HPCC Group Grant NAG5-2218. DAJ is supported at LPTHE by an EEC HCM fellowship, EEC HCM network grant and an Alliance grant. Some of the analysis of Binder's cumulant was carried out using programs written by Andre Krzywicki, whom DAJ would also like to thank for useful discussions. The simulations reported in this work were carried out on the Edinburgh Concurrent Supercomputer (a Meiko Computing Surface) while one of us (KAH) was employed by the Edinburgh Parallel Computing Centre. These simulations used in excess of 32,000 T800 transputer-hours of computation time. 


\section{References}

[1] V.G. Knizhnik, A.M. Polyakov and A.B. Zamolodchikov, Mod. Phys. Lett. A3 (1988) 819;

F. David, Mod. Phys. Lett. A3 (1988) 1651;

J. Distler and H. Kawai, Nucl. Phys. B321 (1989) 509.

[2] E. Brezin, C. Itzykson, G. Parisi and J.B. Zuber, Commun. Math. Phys. 59 (1978) 35;

M.L. Mehta, Commun. Math. Phys. 79 (1981) 327;

M. R. Douglas and S.H. Shenker, Nucl. Phys. B335 (1990) 635;

D. Gross and A. Migdal, Phys. Rev. Lett. 64 (1990) 127;

E. Brezin and V. Kazakov, Phys. Lett. B236 (1990) 144.

[3] V.A. Kazakov, Phys. Lett. A119 (1986) 140;

D.V. Boulatov and V.A. Kazakov, Phys. Lett. B186 (1987) 379.

[4] For a review see: C. Itzykson and J.-M Drouffe, "Statistical Field Theory", (Cambridge University Press, 1989).

[5] C. Baillie and D. Johnston, Mod. Phys. Lett. A7, 1519 (1992); Phys. Lett. B286, 44, (1992).

[6] Z. Burda and J. Jurkiewicz, Acta Physica Polonica B20 (1989) 949. J. Jurkiewicz, A. Krzywicki, B. Petersson and B. Soderberg, Phys. Lett. B213 (1988) 511;

R. Ben-Av, J. Kinar and S. Solomon, Nucl. Phys. B ( Proc. Suppl.) 20 (1991) 711;

S. Catterall, J. Kogut and R. Renken, Phys. Rev. D45, 2957, (1992); Phys. Lett. B292, 277, (1992);

J. Ambjørn, B. Durhuus, T. Jonsson and G. Thorleifsson, Nucl. Phys. B398, 569, (1993).

[7] M. Bowick, M. Falcioni, G. Harris and E. Marinari, "Two Ising Models Coupled to 2 Dimensional Gravity", SU-HEP-4241-556, hep-th/9310136; "Critical Slowing Down of Cluster Algorithms for Ising Models Coupled to 2-d Gravity", SU-HEP-4241-560, hep-lat/9311036.

[8] D. Espriu, M. Gross, P.E.L. Rakow and J.F. Wheater, Nucl. Phys. B265 (FS15) (1986) 92; J.B. Zhang and D.R. Ji, Phys. Lett. A151 (1990) 431.

[9] V. Dotsenko and V. Dotsenko, Adv. Phys. 32 (1983) 129.

[10] W. Janke, M. Katoot and R. Villanova, Phys. Lett. B315 (1993) 412.

[11] M.E. Agishtein and C.F. Baillie, Mod. Phys. Lett. A6 (1991) 1615.

[12] M.E. Agishtein and A.A. Migdal, Nucl. Phys. B350 (1991) 690.

[13] H. Kawai, Y. Kitazawa and M. Ninomiya, Nucl. Phys. B393 (1993) 290.

[14] H. Kawai, N. Kawamoto and Y. Watakibi, Phys. Lett. B306 (1993) 19.

[15] S.F. Edwards and P.W. Anderson, J. Phys. F5 (1975) 965;

M. Mezard, G. Parisi and M. Virasoro, "Spin Glass Theory and Beyond" World Scientific, 1987;

K. Binder and A.P. Young, Rev. Mod. Phys. 58 (1986) 801.

[16] D. Johnston, Phys. Lett. B277 (1992) 405.

[17] V.I. Dotsenko and V.A. Fateev, Nucl. Phys. B240 (1984) 312, B251 (1985) 691.

[18] G. Harris, "Percolation on Strings and the Coverup of the C=1 Disaster", Syracuse preprint SUHEP-4241-555, hep-th/9310137.

[19] J. Wheater, Phys. Lett. B198 (1987) 373;

J. McCarthy, Nucl. Phys. B275 (1986) 421. 


\section{Figure Captions}

Fig. 1. The maximum in the slope of Binder's cumulant is plotted against the lattice size to extract $\nu D$. The best fit is shown as a line.

Fig. 2. The $\beta$ value of the maximum in the slope of Binder's cumulant and the peak in the specific heat are plotted against $N^{-1 / \nu D}$ to extract the critical coupling $\beta_{c}^{\infty}$, given by the intersection of the best fit lines with the $y$ axis.

Fig. 3. A finite size scaling plot of $\chi$, with lines of slope 1.75 (the steeper) and 2 added for comparison. 
This figure "fig1-1.png" is available in "png" format from: http://arxiv.org/ps/hep-lat/9401023v1 
This figure "fig1-2.png" is available in "png" format from: http://arxiv.org/ps/hep-lat/9401023v1 
This figure "fig1-3.png" is available in "png" format from: http://arxiv.org/ps/hep-lat/9401023v1 